\title{
Igor Panasiuk
}

ORCID: 0000-0002-0121-8058

Jakob-von-Paradies-Akademie, Gorzów Wielkopolski

https://doi.org/10.19195/0435-5865.145.15

\section{Das freie assoziative Experiment als psycholinguistische Untersuchungsmethode des Übersetzungsprozesses: Ein theoretischer Exkurs}

\begin{abstract}
s
Der Artikel handelt von der psycholinguistischen Methode der Untersuchung des kognitiven Prozesses der Textrezeption und des Übersetzungsprozesses - dem freien assoziativen Experiment. Das psycholinguistische Experiment kann als eine komplexe Untersuchungsmethode angesehen werden. Die Anwendung des psycholinguistischen Experiments ist in das translatorische Experiment im Aspekt der Polyvarietät der Übersetzung eingebettet: Es werden zwei deutsche Übersetzungen des Romans „Der Meister und Margarita“ von Michail Bulgakow und des Romans „Doktor Shiwago" von Boris Pasternak im Rahmen des psycholinguistischen Experiments analysiert. Die Quelle für die Polyvarietät der Übersetzung ist der subjektive Charakter der Bedeutungsinterpretation, die auf den probabilistischen Prozessen, denen assoziative Bedeutungen zu Grunde liegen, basiert. Emotionen spielen hierbei auch eine wichtige Rolle. Die gewonnenen empirischen Daten können zu didaktischen Zwecken bei der Ausbildung von angehenden Translatoren eingesetzt werden.
\end{abstract}

Schlüsselwörter: freies Assoziieren, Translationsprozess, psycholinguistische Untersuchungsmethode, Wahrscheinlichkeitsprognose, Inferenz, Abduktion, Implikation, probabilistische Prozesse, Lakune, Polyvarietät der Übersetzung

\section{The free associative experiment as a psycholinguistic investigation method of the translation process: A theoretical insight}

This paper deals with the psycholinguistic methods of investigation of the cognitive process of text reception and the process of translation - the free associative experiment. The psycholinguistic experiment can be seen as a complex investigation method. The application of the psycholinguistic experiment is thus to be embedded in the translational experiment in the aspect of the polyvariety of translation: Two German translations of the novel The Master and Margarita by Mikhail Bulga- 
kov and the novel Doctor Zhivago by Boris Pasternak are to be analyzed in the frames of the psycholinguistic experiment. The source of the polyvariability of translation is the subjective character of the interpretation of meaning, which is based on probabilistic processes and associative meanings. Emotions play an important role here. The empirical data obtained will be used for didactic purposes in the training of prospective translators.

Keywords: free associative experiment, translation process, psycholinguistic investigation method, anticipation, inference, abduction, implication, probabilistic process, lacuna, polyvariety of translation

Igor Panasiuk, Zakład Języka Niemieckiego, Wydział Humanistyczny, Akademia im. Jakuba z Paradyża w Gorzowie Wielkopolskim, ul. Teatralna 25, 66-400 Gorzów Wielkopolski, Polen, E-Mail: igor_panasiuk@wp.pl

Received: 19.10.2019, accepted: 8.04.2020

\section{Theoretische Grundlagen der Studie}

Im vorliegenden Beitrag handelt es sich um die Bewährungsprobe für die Hypothese, den Übersetzungsprozess mit Hilfe des freien assoziativen Experiments unter anderem für didaktische Zwecke zu untersuchen. Die Anwendung des freien assoziativen Experiments zur Beschreibung des Übersetzungsprozesses wurde bisher im Rahmen der prozessorientierten Übersetzungswissenschaft nicht unternommen.

Im idealtypischen Modell des Übersetzungsprozesses, das den zentralen Aspekt seiner Konzeption des Konstruktiven Übersetzens darstellt, spricht Hans G. Hönig (1995: 40ff.) vom Erfassen des ausgangssprachlichen Textes durch den Übersetzer, der als erstes diesen Text aus seiner ,natürlichen“ Umgebung in der realen Kommunikation entfernt und in die eigene mentale Realität projiziert. Das Projizieren des zu übersetzenden Textes in die eigene mentale Realität erfolgt durch das Einschalten des mentalen Lexikons des Übersetzers, in dem der projizierte ausgangssprachliche Text zum Objekt seiner mentalen Verarbeitungsprozesse wird. Die Rezeption jedes Textes involviert somit eine sofortige Aktivierung des mentalen Lexikons des Textrezipienten. Die kognitiven Verarbeitungsprozesse des mentalen Lexikons sind Mechanismen des Verstehensprozesses, in dem sich unwillkürlich Erwartungsstrukturen probabilistischen Charakters in Bezug auf die Gesamtgestalt und den gesamten Textinhalt aufzubauen beginnen. Das mentale subjektive Lexikon betrachtet Kiss (1968) als System der Informationssuche, das sich auf stochastische Prozesse gründet. Eine solche Betrachtungsweise der Spezifik des mentalen Lexikons wird dem System einer passiven Aufbewahrung der Information entgegengestellt und soll den Blick auf die Notwendigkeit verschärfen, die stochastischen Prozesse der Informationsverarbeitung durch einen Menschen zu berücksichtigen. Kiss ist dabei der Meinung, dass das Lexikon eines Menschen die 
Repräsentation von Wörtern bzw. von größeren verbalen Einheiten beinhaltet, die über mehr als eine Bedeutung verfügen können. Jede der Bedeutungen des jeweiligen Wortes ist aber mit einem Begriff verbunden. Was die Struktur betrifft, so stellt das Lexikon ein Netz von verbalen Repräsentationen und Verbindungen zwischen ihnen dar. Jedes Wort bzw. seine Repräsentation zeichnet sich durch eine variierende Stufe der Aktivität aus. Die Verbindungen leiten diese Aktivität von einem Wort zu dem anderen weiter. Dabei ist jedes Wort im Stande, eine summarische Aktivität, die binnen einer bestimmten Zeitspanne vom Wort erhalten worden ist, zu akkumulieren. Deswegen kann das Funktionieren des Lexikons am besten mit Hilfe eines stochastischen, sich verästelnden Prozesses beschrieben werden. Die Übergänge, die durch Veränderungen auf den Ebenen der Aktivität von Wörtern bedingt sind, können von folgenden Faktoren verursacht werden: 1. Information, die mit Hilfe von Wahrnehmungsmechanismen über Gefühlsorgane erhalten wird; 2. Denkprozesse; 3. die innere Wechselwirkung von Einheiten eines Lexikons, wenn sich die Systemordnung beim Fehlen eines äußeren Einflusses ändert. Kiss betont dabei den dritten Typ der Übergänge und bezeichnet sie als „frei“. Sie seien ihrer Natur nach stochastisch, denn die Aktivität des einen Wortes könne unter bestimmter Wahrscheinlichkeit durch die Aktivität des anderen Wortes verändert werden. Betrachtet man die möglichen physiologischen Mechanismen des Funktionierens dieses Systems, so stellen die Wortrepräsentationen eine Auswahl von Neuronen dar. Da die Neuronen durch Synapsen miteinander verbunden sind, beeinflusst der Reiz eines Neurons unter bestimmter Wahrscheinlichkeit die geordneten Reihen der anderen Neuronen (Kiss 1969b, siehe: Zalevskaja 2005: 153). Die beiden Aspekte - die psycholinguistische Organisation von Bedeutungen im mentalen Lexikon sowie ihre neuronale Repräsentationen - liefern in ihrer Verbindung eine Vorstellung über den Ablauf des Verstehens- und Übersetzungsprozesses, die einem Übersetzer das bewusste Ausüben seiner Tätigkeit steigert.

Die Nennung eines verbalen Stimulus bei der Durchführung eines freien assoziativen Experiments gibt eine bestimmte Aktivitätsstufe der Repräsentation eines oder mehrerer Wörter im System vor. Das erweist sich als Anstoß für die Veränderung der Systemordnung über die freien Übergänge, die zur Veränderung von Aktivitätsstufen von Systemelementen beitragen. In einem bestimmten Moment trifft ein Proband eine Wahl für ein Assoziat, die durch eine relative Aktivität des letzteren bestimmt ist. Da sich das Entscheidungstreffen durch einen stochastischen Charakter auszeichnet, variiert die Wahl der Assoziation, was die Möglichkeit für die Polyvarietät der Übersetzung bietet (Panasiuk 2016). Wenn dieses Experiment mit einer Probandengruppe durchgeführt wird, bestimmt eine Reihe von Faktoren die verschiedene Basisstruktur der Übergänge zwischen den Systemelementen. Trotz alledem wird eine erhebliche Affinität in den assoziativen Netzen verschiedener Probanden festgestellt (Kiss 1969a, Zalevskaja ebd.). Diese Tatsache spricht eindeutig und überzeugend für die Durchführung des freien assoziativen Experiments mit mehr als einem Probanden (Übersetzer) zwecks Un- 
tersuchung des Verstehens- und Übersetzungsprozesses. Letzteres rechtfertigt die Durchführung des translatorischen Experiments von der Außenperspektive (retrospektive Sicht auf den Übersetzungsprozess) sowie von der Innenperspektive (introspektive bzw. prospektive Sicht auf den Übersetzungsprozess) mit mehreren Übersetzern zwecks einer hypothetischen Rekonstruktion des Übersetzungsprozesses (Panasiuk 2016).

Der von Kiss und seinem Team zusammengestellte „Assoziative Thesaurus der englischen Sprache“ lässt über den Charakter und die Kraft von hinausgehenden (von Stimulus zu Reaktion) sowie den hereinkommenden Verbindungen (von Reaktion zu Stimulus) zwischen den untersuchten Wörtern urteilen. Das Vorhandensein von Informationen über die rücklaufenden Assoziationen im assoziativen Thesaurus, d.h. darüber, welche Wörter ein bestimmtes Wort als Reaktion auslösen können, gibt einen prinzipiell neuen Aufschluss über die assoziative Struktur des mentalen Lexikons. Laut Kiss (1973: 5ff.) gelten die verbalen Assoziationen als unmittelbare Indikatoren des Verbindungsgrades zwischen den Begriffen. Deswegen geben die assoziativen Normen sowie der assoziative Thesaurus eine anschauliche Kartierung dieses Aspektes unseres Wissens. Die Möglichkeit dieser Information ist über jeden Zweifel erhaben, denn die assoziative Organisation gilt als Aspekt vieler, wenn nicht aller kognitiven Prozesse, die den Einsatz des subjektiven Lexikons verlangen. In dem von Kiss entwickelten Modell des Lexikons geht der Autor von den Erkenntnissen der modernen Neurophysiologie aus und konstruiert ein Modell des Lexikons unter Berücksichtigung von probabilistischen Gesetzmäßigkeiten, die beschreiben, wie das Gedächtnis eines Menschen funktioniert. Bei dem im assoziativen Thesaurus präsentierten Material geht es um assoziative Felder, die im Vergleich zu den semantischen Feldern viel breiter angelegt sind. Ein assoziatives Feld ist als Netz von Assoziationen strukturiert. Anderson und Bower (1973) gehen dabei davon aus, dass sich Wörter gegenseitig assoziieren können, wenn die ihnen entsprechenden Begriffe Bestandteil der im Gedächtnis kodierten Propositionen sind. Ihrem Inhalt nach gibt eine Proposition eine bestimmte Aussage über die Außenwelt wieder. Von daher stellt das menschliche Langzeitgedächtnis ein riesiges Netz von sich gegenseitig überschneidenden propositionalen Verästelungen dar. Jede der Verästelungen schließt eine bestimmte Auswahl an Gedächtnisknoten mit markierten Verbindungen ein. Anderson (1976) führt weiterhin aus, dass jeder Knoten des propositionalen Netzes einen Begriff bzw. ein Konzept präsentiert. Mit diesem Knoten sind alle mit dem Konzept verknüpften Informationen verbunden, was dem propositionalen Netz die Eigenschaft vermittelt, die Anderson als Indexierung über einen Begriff bezeichnet: wenn ein Begriff im System des Gedächtnisses geortet werden kann, so können dort auch alle in diesem Zusammenhang uns bereits bekannten Informationen gefunden werden.

Eine ähnliche Sicht auf das mentale Lexikon liefert auch Osgood (1957, 1980), bei dem es als einer der wichtigsten Mechanismen der kognitiven Informa- 
tionsverarbeitung gilt. Das mentale Lexikon ist laut Osgood eher ein Prozess als ein Informationsspeicher, gilt als eine große Auswahl an Verbindungen zwischen Zeichen (Perzepten) und Kodes semantischer Merkmale. Die semantischen Merkmale betrachtet Osgood als mediative (d.h. vermittelnde) Komponenten, die ihrer Natur nach bipolar sind und über eine variierende Größe der Intensität zwischen Null (Neutralität, das Fehlen eines Merkmals in der Kodeskette) und einen maximalen Nenner auf der Skala verfügen. Eine signifikative Rolle spielt dabei die Notwendigkeit einer emotional-axiologischen Einstellung zu den Trägern dieser Merkmale. Ins Spiel kommt hier bereits der Einfluss von Emotionen auf die Kognition eines Menschen.

Das Erfassen des Sinns des zu übersetzenden Textes ermöglicht somit die probabilistische Struktur der Sprache. Die Sinnerkennung bei der Textrezeption erfolgt somit durch den Mechanismus der Wahrscheinlichkeitsprognose, die auf der Basis der assoziativen Bedeutung funktioniert. Hörmann (1967: 185) beruft sich dabei in seiner Monographie „Psychologie der Sprache“ auf Deese (1962: 163), der die Bedeutung eines Wortes als Verteilung der Wort-Responses definiert, die auf der Grundlage eines verbalen Stimulus, der als ein zu vergleichendes Wort auftritt, produziert werden. Bedeutung ist in diesem Zusammenhang ein Netz verbaler Assoziationen. Hörmann führt weiterhin sich auf Deese stützend aus, dass je größer die assoziative Bedeutungs $\neg$ ähnlichkeit zweier Wörter sei, desto größer die Wahrscheinlichkeit sei, dass diese Wörter in derselben situativen und verbalen Umgebung vorkämen. Und umgekehrt: Je mehr Satzrahmen (frames) es gebe, in denen statt eines Wortes ein anderes Wort gebraucht werden könnte, desto größer würden die beiden Wörter in ihrer Bedeutung Ähnlichkeiten aufweisen. Auf der Grundlage der assoziativen Ähnlichkeit verläuft die Entwicklung des Kontextes vom Wort (Stimulus) zum Response hin (verbale Assoziation auf diesen Stimulus). Deese (ebd.) versucht dabei die assoziative Bedeutung zu erfassen, indem er die Relationen beschreibt, die zwischen den Responses auf verschiedene Stimuli bestehen. Er geht davon aus, dass zwei Stimuli sich in ihrer assoziativen Bedeutung in dem Maße ähnlich sind, in dem sich die Verteilungen ihrer Assoziationen gleichen. Der Zusammenhang mit und zwischen den assoziativen Responses ist somit die assoziative Bedeutung des betreffenden Stimulus. Dies ist übrigens eine der Prämissen für die Polyvarietät der Übersetzung, denn jede der Responses, die eine assoziative Relation zu einer anderen Response aufweist, kann auch als eine der Varianten für den betreffenden Stimulus auftreten. Denn die Synonymie stellt bereits eine Response für einen bestimmten Stimulus dar. Assoziative Bedeutung, die auch der Synonymie zu Grunde liegt, stellt auch eine Basis für die Entwicklung von varianten Relationen zwischen den zu vergleichenden Stimuli dar. Die assoziative Bedeutung ist demzufolge aktualisierte kontextuelle Bedeutung, denn durch Assoziationen entstehen Relationen zwischen Stimulus und Response, die im Rahmen bereits bestehender Kontexte vorhanden sind. Die Funktion des Mechanismus der Wahrscheinlichkeitsprognose kann daher am Mechanismus der 
Assoziation veranschaulicht werden. Denn wie Hörmann (1967: 85f.) behauptet, brauche man für die Zahlen wenige akustische Charakteristika, um feststellen zu können, ob es um eine bestimmte Zahl gehe. Bei einem sinnvollen Wort ist der Hörer schon auf mehrere akustische Kennzeichen angewiesen, um es empfangen zu können. Würden z.B. einige dieser Zeichen im Rauschen untergehen, so wird er die fehlenden Kennzeichen aus einem Wissen über die kontextuelle Situation (top-down-Prozesse), aber auch über die Buchstaben- und Wortkombination, die in der jeweiligen Sprache üblich ist, ergänzen können. Dem Kontext wird hier die vorrangige Rolle zuerkannt, denn die isolierten Wörter würden bei einem bestimmten Signal-Rauschen-Abstand schlechter erkannt werden als dieselben Wörter bei gleichem Rauschen in Sätzen, d.h. im Kontext bzw. Kotext, „denn aus dem Bau des Satzes ergeben sich ja auch Hinweise auf das, was als Wort an dieser bestimmten Stelle möglich ist" (Hörmann 1967: 86).

Hörmann (ebd., S. 87) spricht hierbei von der Auftretenswahrscheinlichkeit der Wörter im Kontext, die auf die Länge der Nachricht und im Zusammenhang damit die Nachricht-Einheiten sowie die Anzahl von Möglichkeiten pro Einheit ankommt. Gemünzt auf die Situation des Simultandolmetschens kann der Informationsverlust von diesen Faktoren abhängen, wobei pro Nachrichten-Einheit umso mehr Information verloren geht, je größer die Anzahl der Einheiten in der Nachricht und je größer die Anzahl der Möglichkeiten pro Einheit ist (ebd.). Die Auftretenswahrscheinlichkeit der Wörter ist auch kontextuell bedingt und wird auch mit der Sprache erlernt. Sie bildet somit das Wahrscheinlichkeitsprofil einer Sprache heraus (Hörmann ebd., S. 87). Der menschliche Organismus ist stets danach bestrebt, die durchschnittliche Anstrengung in der Zeit möglichst gering zu halten. Diesem Prinzip unterliegt auch nach Hörmann (ebd., S. 89) die Worthäufigkeit in der Sprache: „In der Sprache stehen sich zwei Tendenzen gegenüber: eine Tendenz, sich kurz zu fassen, und eine Tendenz, sich verständlich zu machen." Der Wahrnehmungsvorgang sprachlicher Ereignisse, der Akt des Erlernens, im Gedächtnis ablaufende psychische Ereignisse werden laut Hörmann (ebd.) mit der Häufigkeits- bzw. Wahrscheinlichkeitsstruktur der sprachlichen Welt in Verbindung gebracht. Der Zuordnungsschritt bei der Wahl einer Übersetzungsvariante wird in Anlehnung an Hörmann (ebd., S. 97) nicht dem argumentativen Gewicht von tatsächlich aufgefassten Eigenschaften des Objektes Vorrang geben, sondern die Klasse der Eigenschaften des Objektes tritt dabei als die treibende Kraft auf, die einen „Sog“ ausübt, der das Objekt in diese Klasse, d.h. das kontextuell abgegrenzte semantische Feld, hineinreißt. Die Kraft dieses Sogs ist umso stärker, je häufiger diese Klasse vorher in der Sprache vorgekommen ist. Die Wahrscheinlichkeitsstruktur einer Sprache tritt dabei in ihrer einfachsten Form als Durchschnittswerte eines Auftretens bestimmter Einheiten zum Vorschein, die über Millionen von Wortsequenzen gesammelt worden sind. Es geht dabei nicht ums Aufdecken von probabilistischen Gesetzmäßigkeiten einer ganzen Sprache, sondern um die probabilistischen Gesetzmäßigkeiten des einzelnen Spracher- 
eignisses, was im Falle eines Übersetzungsaktes z.B. die Übersetzungseinheit sein kann (Hörmann ebd., S. 101).

Der Verlauf des Mechanismus der Wahrscheinlichkeitsprognose erfolgt durch die Auslösung von syntagmatischen und paradigmatischen Assoziationen. Die Dimension der Ähnlichkeit von Bedeutungen gestaltet sich durch die Annäherungsgrade an echte Sprache, wobei im Falle des Verstehens- und Übersetzungsprozesses immer nach syntagmatischen und paradigmatischen Bedeutungen gegriffen wird. Bei den syntagmatischen Assoziationen handelt es sich um die kontextuellen Verbindungen zwischen den Wörtern innerhalb eines Satzparadigmas, während es bei den paradigmatischen Assoziationen um die Suche nach kontextuellen Relationen der Synonymie geht: „Paradigmatische Assoziationen kommen zustande dadurch, dass der Stimulus ein Wort ist, das an Stellen vorkommt, für die der Hörer dieses und einige Wörter bereitgestellt hat. Assoziationen auf ein bestimmtes Stimulus-Wort werden also (zumindest weitgehend) von dem Kontext determiniert..."(Hörmann 1967: 144).

Assoziativen Beziehungen liegen bestimmte Wahrscheinlichkeitsbeziehungen zwischen Stimulus und Response zu Grunde. Von den Wahrscheinlichkeitsbeziehungen werden auch im Falle des Übersetzens die Annäherungsgrade an das Original geprägt. Die Bedeutung eines Wortes hängt somit laut Hörmann (ebd., S. 146) mit den Assoziationen zusammen, die das betreffende Wort auslöst. Der Kontext eines Wortes kann die Bedeutung dieses Wortes dadurch beeinflussen, dass durch diesen Kontext bestimmte Assoziationen dieses Wortes den anderen überlegen sind, die momentan in den Hintergrund treten und weniger verfügbar werden (ebd., S. 147).

Die assoziative Betrachtungsweise beschäftigt sich also Hörmann (ebd., S. 116) zufolge nicht mit den Verhältnissen zwischen Einheiten, die in einem konkreten Sprachereignis vorkommen, sondern mit einer manifesten Einheit und einer oder mehreren latenten, d.h. nicht ausgesprochenen Einheiten. Daher erfolgt die Wahrscheinlichkeitsprognose in erster Linie durch die Herstellung von assoziativen Relationen zwischen den bereits vorhandenen Wörtern (Stimuli) und ihren assoziativen Responsen. Eine assoziative Relation ist die Relation auf kognitiver Ebene des Kontextes, die in seiner Tiefenstruktur im Verborgenen liegt. Im Unterschied zur syntaktischen Relation, die als Relation in praesentia gilt, vereinigt die assoziative Relation laut de Saussure Begriffe in absentia zu einer potentiellen Reihe (ebd.). Mit Hilfe des psycholinguistischen assoziativen Experiments kann das Funktionieren des Mechanismus der Wahrscheinlichkeitsprognose in einer konkreten Wortsequenz demonstriert werden.

Die Wahl von Assoziationen als Denkoperation verläuft A.N. Leont'ev (1969/1975: 13) zufolge nach einem Plan, der sich aus bestimmten Einheiten zusammensetzt, die als Regeln für die Organisation des Verhaltens bezeichnet werden können. Bei der Ausführung dieses Plans kann es zu Störungen kommen, die Ejger (1990: 76) durch die Störung der Verbindung der Kontrolle zwischen den 
Hemisphären des Gehirns erklärt. Der Mechanismus des Ins-Schwarze-Treffens verläuft durch die Wechselwirkung zwischen den Blöcken des Entscheidungstreffens der linken und rechten Hemisphäre im Gehirn. Die sprachlichen Strukturen, nachdem sie erkannt worden sind, werden mit den im Gehirn bereits vorhandenen Strukturen verglichen, indem sie vom Block des Entscheidungstreffens in die Vergleichsvorrichtung überführt werden (ebd., S. 77). Zalevskaja (1978: 73) merkt an, dass wenn nach einem notwendigen Wort oder einem Begriff im Gedächtnis gesucht wird, sich der Mensch längs der assoziativen oder logischen Strukturlinien, die das Organisationssystem des ganzen Wortschatzes durchbohren, bewegt. Die Wahl einer Variante erfolgt Ejger (ebd., S. 80) zufolge, das Treffen ins Schwarze, durch die Vereinigung der konsequenten und parallelen Vergleichsaktionen. Die Erkennungsaufgabe wird durch das konsequente Aktivieren der Blöcke gelöst, wobei jeder der Blöcke nach dem Prinzip der Parallelität des Vergleichs funktioniert. Eine verhältnismäßig geringe Anzahl von Mustern innerhalb jedes Blocks schafft günstige Konditionen für ihr gleichzeitiges Einschalten im Vergleichsprozess. Diese parallel ablaufenden Prozesse ermöglichen es, bereits in der ersten Etappe, das Objekt einer bestimmten Klasse zuzuordnen und damit die erste Entscheidung zu treffen.

Der probabilistische Mechanismus des Assoziierens kann auch durch die Art und Weise des Inferierens, also durch Inferenzen, das heißt durch Implikationen und zum Teil auch in Verbindung mit Abduktionen erklärt werden. Die Wahrscheinlichkeitsprognose setzt sich aus zahlreichen Inferenzprozessen zusammen. Die beiden interpretativen Verfahren - Implikation und Abduktion, durch die das Verweisen der zu übersetzenden Zeichen auf ihre Interpretanten im Prozess der Semiose, d.h. ihre Interpretation, stattfindet, sind laut Siever (2010: 202) die grundlegenden Verfahren beim Verstehen und Übersetzen. Die beiden Verfahren können auch als kognitive Mechanismen des Übersetzungsprozesses angesehen werden. Übersetzungen sind also Interpretationen des Ausgangstextes, wobei jede Interpretation eine Übersetzungsvariante des Originals darstellt. Wenn zwischen dem Ausgangs- und Zieltext ein Implikationsverhältnis vorliegt, so hält Siever (ebd., S. 320) den Zieltext für den Interpretanten des Ausgangstextes. Die Übersetzungsvariante ist also der Interpretant des Originalzeichens. Abduktion liefert immer nur mögliche Inferenzen, wogegen die Induktion wahrscheinliche und die Deduktion notwendige Inferenzen liefern (Gorlée 2000: 163). Abduktion ist Gorlée (ebd.) zufolge eine Erklärungsmethode zwecks Erzeugung einer annehmbaren Hypothese: „Abduction is an exploratory method to create a simple and attractive hypothesis which accounts for the external experience under investigation" (Gorlée 2004: 118).

Chernov (1990: 132) spricht in diesem Zusammenhang von Quellen und Grenzen der Varietät in der Übersetzung, indem er das Verstehen der Botschaft in der Herstellung von Inferenzen über die Bedeutung dieser Botschaft sieht. Es geht dabei um die Herstellung nicht nur einer Inferenz, sondern mehrerer Inferenzen, die linguistisch, kognitiv, deiktisch (oder situativ) und pragmatisch sein können. 
Der Verlauf der Wahrscheinlichkeitsprognose (,a decisive factor of choice between possible alternative ways of understanding the utterance“) wird laut Chernov (ebd.) hauptsächlich von der Wahl der Situation (also von der kontextuellen Wahl, ,verbal contextual situation, or verbal context, of the utterance“) bestimmt, und sobald diese Wahl getroffen worden ist, entscheidet sich der Übersetzer, die jeweilige Bedeutung der zu übersetzenden Äußerung anzunehmen. Die Länge des Kontextes entscheidet dabei über den Erfolg der Disambiguierung der im Übersetzungsprozess zu erschließenden Bedeutung und damit über den Erfolg des Verlaufs der Wahrscheinlichkeitsprognose schlechthin: Unterschiedliche Äußerungen benötigen laut Chernov (ebd.) unterschiedliche Längen des Kontextes, damit sie erfolgreich disambiguiert werden können. Wenn der Übersetzer mit einem Ausgangstext aus einer anderen Kultur oder Epoche zu tun hat, erweisen sich für ihn die kognitiven Inferenzen als weniger produktiv, dafür aber werden die deiktischen bzw. situativen Inferenzen an Bedeutung der Wahrscheinlichkeit (,a matter of probabilities") gewinnen. Sollte es der Übersetzer mit einem Text eines unbekannten Autors zu tun haben, bleiben die pragmatischen Inferenzen unbestimmt. Chernov (1990: 133) fasst an dieser Stelle zusammen: Die meisten Quellen für die Variabilität sind linguistischer und kognitiver Natur, während die Grenzen der Variabilität und die aktuelle Wahl von möglichen Alternativen beim Verlauf der Wahrscheinlichkeitsprognose von situativen Inferenzen bestimmt werden. Die Wahrscheinlichkeitsprognose wird somit durch die Herstellung der situativen Inferenz durch den Kontext hindurch weiter geführt. Gerade die situative Inferenz bestimmt über die entschiedene Begrenzung der Variabilität in der ersten Phase, die in die zweite Phase wechselt, damit ein vollständiges Bild von Quellen und Grenzen der Variabilität im Translationsprozess beim Übersetzer entstehen kann.

Das Situative bzw. Kontextuelle an der referenziellen Bedeutung zu erkennen, heißt Chernov (ebd., S. 134) zufolge, die Funktion der minimalen Länge des Kontextes bestimmen zu können, womit im Grunde genommen eine Übersetzungseinheit bestimmt werden kann. Dieser kognitive Vorgang fußt auf der situativen Inferenz. Die Hauptsache dabei ist, den richtigen Referenten sowie die richtigen Kriterien für die Bestimmung des minimalen Kontextes zu finden, der für den Verlauf der Antizipation unabdingbar ist. Durch Abduktion werden somit die mentalen und nicht direkt zugänglichen, in der black box befindlichen Prozesse beim Übersetzen greifbar (Gorlée 2000: 169). Die Wahrscheinlichkeitsprognose zeichnet sich daher durch den abduktiv-induktiven Charakter aus. Antizipationen sind laut Serson (2000: 278) probabilistische oder wahrscheinliche Schlussfolgerungen, also die Schlussfolgerungen der Induktion und Abduktion. Černov (1987: 19) sieht weiterhin im stochastischen bzw. wahrscheinlichen Charakter der Implikation das Gesetz der Vielfältigkeit von Übersetzungsvarianten, das vorsieht, dass die Übersetzung jeglichen Textes nicht in einer, sondern in mehreren Varianten möglich ist. Černov (ebd.) sieht das Verstehen einer Redemitteilung sowohl als Prozess als auch als Ergebnis von Sinnschlussfolgerungen an, wobei die gehörte Mitteilung 
mit der semantisch-inhaltlichen Struktur der Mitteilung als sprachliche Schlussfolgerung (a), mit Inhaltskomponenten des eigenen Langzeitgedächtnisses (Wissen über die Welt) als Thesaurusschlussfolgerung (b), mit Faktoren des situativen Kontextes als situativ-deiktische Schlussfolgerung (c) sowie mit sozialen und rollenspezifischen Charakteristika des Sprechenden und des Adressaten als pragmatische Schlussfolgerung verglichen wird. Da Implikation immer Differenz ergibt, kennzeichnet die Letztere den Abstand der Übersetzungsvariante zum Original. Die Differenz kommt somit in der Struktur der denotativen Bedeutung der Übersetzungsvariante, die als Resultat der Implikation gilt, zum Ausdruck und kann mit der semantischen Intensität der Lakunen gemessen werden. Die Klassifizierung der Polyvarietät der Übersetzung kann sowohl nach der Art der Implikation als auch nach der semantischen Intensität der Lakune erfolgen. Die Differenz entsteht somit infolge des wahrscheinlichen Charakters des Implikationsverhältnisses.

Die Wahrscheinlichkeitsprognose auch unter Aktivierung des Mechanismus des code-switching wird bereits beim Lesen des zu übersetzenden Textes durchgeführt, worauf auch Umberto Eco (1994: 143) hinweist. Bei einem weiteren Verlauf der Fabel vollzieht der Leser implizite Vorhersagen bzw. Antizipationen, die die logische Form des abduktiven Schlusses (der abduktiven Inferenz) annehmen. Das lässt Eco schlussfolgern, dass die Antizipationen des Lesers an der Fabel teilhaben, was im Falle der Übersetzung die Interpretation der Fabel durch den Übersetzer etabliert. Die Antizipation kann somit als semiotisch-kognitiver Mechanismus des Übersetzungsprozesses aufgefasst werden, der nach Gorlée (1994: 226) auf dem Prinzip der Semiotranslation, der Übersetzungssemiose, basiert. Die Übersetzung ist für Gorlée (1994: 212) die konventionelle Semiose (,contractual semiosis“), deren konventioneller Charakter psychologischer Natur ist und in der Wahrscheinlichkeit der Konvention zum Ausdruck kommt. Die Konventionalität der Semiose bestimmt nämlich die Übersetzbarkeit. Daher kann es keine statische Beschreibung der Semiose geben. Das prägt auch die Beschreibung des Translationsprozesses in der Hinsicht, dass der Letztere nur hypotetisch rekonstruiert werden kann.

In didaktischer Hinsicht erweist sich die Anfertigung einer Liste von Inferenzen bzw. Infenrenztypen als wichtig, die im Übersetzungsunterricht eingeübt werden können. Die abduktive Kompetenz stellt laut Siever (2010: 319) überdies den Kern der kommunikativen Kompetenz dar und macht ein kreatives Übersetzen im Sinne von Kußmaul möglich. Die Prozesse der Inferenz, Abduktion und Implikation sind erlernbar und können im Übersetzungsunterricht trainiert werden. Nach Inferenzprozessen, aus denen sich die Antizipation zusammensetzt, kann über die Organisation der inneren Struktur des mentalen Lexikons des Übersetzers geurteilt werden. Sie verlaufen im Bewussten, sind daher bewusst greifbar und können demzufolge für die Translationsdidaktik fruchtbar gemacht werden. Das psycholinguistische Experiment und vor allem das assoziative Experiment leisten beim Aufdecken dieser kognitiven Prozesse eine enorme Hilfe: Die kogni- 
tiven Prozesse der Antizipation können somit hinter den Relationen zwischen Stimulus und Responses entdeckt werden.

\section{Methodische Vorgehensweise}

Die Methodologie dieser Studie beinhaltet eine Reihe von Aspekten aus dem Bereich der Psycho- und kognitiven Linguistik sowie den kognitiven Neurowissenschaften, die die Anwendung der psycholinguistischen Forschungsmethode - das psycholinguistische Experiment - theoretisch untermauern bzw. festigen. Unter dem psycholinguistischen Experiment ist eine komplexe Untersuchungsmethode des menschlichen Sprachbewusstseins zu verstehen. Das psycholinguistische Experiment setzt sich aus drei Phasen zusammen, in dessen Mittelpunkt das kontextuell gebundene assoziative Experiment steht. Letzteres eignet sich für die Untersuchung des Übersetzungsprozesses in der Hinsicht, dass damit die assoziative Bedeutung untersucht wird. Laut Hönig (1995: 62) benötigt ein Übersetzer die Assoziationskompetenz, sie sei der eigentliche Motor der Übersetzungsprozesse, das Hauptaggregat im Bereich des unkontrollierten Arbeitsraums. Die Assoziationskompetenz müsse jedoch von einer Makrostrategie gesteuert werden, damit das definierte Ziel der Übersetzung realisiert werden könne. Die Assoziationskompetenz ist zwar nicht der erworbenen Übersetzungskompetenz, die dem kontrollierten Arbeitsbereich angehört, gleichzusetzen, sollte aber von der Letzteren unterstützt werden, damit sie vom Übersetzer bewusst eingesetzt werden könne. Der Übersetzer könne jedoch ohne Makrostrategie, aber mit entsprechend ausgebauter Assoziationskompetenz, besser übersetzen als umgekehrt mit Makrostrategie jedoch mit einer vergleichsweise schwach ausgebildeten Assoziationskompetenz. Der Grund dafür liegt in den am Übersetzungsprozess beteiligten assoziativen Bedeutungen, die als Vehikel für den Verlauf des Kontextes dienen und diesen zu antizipieren ermöglichen.

Den Beweis dafür liefert Paul Kußmaul (2007: 176), indem er unwissentlich das kontextuell gebundene assoziative Experiment bei der Suche nach Übersetzungsvarianten für das englische crime im Satz How successful do you think government in [Country] is nowadays in controlling crime? durchführt. In Folge einer konvergenten Bedeutungsrelation zwischen dem englischen crime und den deutschen Verbrechen und Kriminalität soll also eine Entscheidung für eine der Übersetzungsvarianten getroffen werden. Die Wahl für die Übersetzungsvariante soll nach dem Prinzip der Prototypikalität getroffen werden. Um die prototypischen Szenenelemente der Szene „Verbrechen“ abzurufen, führt Kußmaul die Evaluierung der vorläufigen Übersetzung Was meinen Sie, wie erfolgreich ist heutzutage der deutsche Staat bei der Verbrechensbekämpfung? in Form einer Befragung durch: „Ich sagte also etwa Folgendes: ,Für crime gibt es im Deut- 
schen zwei Entsprechungen: Verbrechen und Kriminalität. Woran denken wir beim Wort »Verbrechen«?"“ Dies ist nichts Anderes als die Situation des freien jedoch kontextgebundenen assoziativen Experiments, in die die Gesprächsteilnehmer durch Paul Kußmaul mit der Frage hineinversetzt werden. Das Wort Verbrechen fungiert hier als Stimulus, für den die Gesprächsteilnehmer die prototypischen assoziativen Bedeutungen nennen sollten. Diese Assoziationen sind mögliche Übersetzungsvarianten, die für den Stimulus crime in Frage kommen könnten, denn ihre Wahl wurde nach dem Prinzip der prototypischen Vertreter der Kategorie „Verbrechen“ getroffen. Daraufhin nannten die Gesprächsteilnehmer folgende Responses „Mord“, „Raub“, „Vergewaltigung“, „Diebstahl mit Körperverletzung", also Sachverhalte, die Gewalt als Haupteigenschaft enthalten. Es sind somit die Kernvorstellungen der Szene Verbrechen. Weiterhin stellte Kußmaul die Frage, ob durch die genannten Assoziationen die Bedeutung von crime in der Übersetzung abgedeckt worden sei, worauf eine spontane Antwort erging, im Ausgangstext sei sicherlich an Wirtschaftskriminalität und alle Arten von Diebstahl, also von Kleinkriminalität gedacht worden. Die Entscheidung wurde endgültig für „Kriminalität“ gefällt und die Übersetzung lautete: Was meinen Sie, wie erfolgreich ist heutzutage der deutsche Staat bei der Bekämpfung von Kriminalität. Das kontextuell gebundene assoziative Experiment erfolgt nach dem Prinzip der Prototypikalität, also der Vergegenwärtigung von Kernvorstellungen im Rahmen eines Kontextes. Assoziative Bedeutungen liegen überdies dem Visualisieren im Übersetzungsprozess zu Grunde, sie stimulieren in Folge ihrer Prototypikalität Szenen, die bestimmte Inhalte visuell im Gedächtnis wachrufen. Dies ist ein Paradebeispiel für die Anwendung des assoziativen Experiments zum Zwecke der Untersuchung und hypothetischen Beschreibung des Übersetzungsprozesses. Diese Tatsache gilt als Begründung für die Durchführung des psycholinguistischen Experiments als Untersuchungsmethode des Übersetzungsprozesses.

Das psycholinguistische Experiment umfasst also drei Schritte: In dem zu übersetzenden Text werden zuerst die lexikalischen Schlüsseleinheiten mit Hilfe der Methode der intuitiven Feststellung von Übersetzungseinheiten nach Wolfram Wilss (1992: 85) ausgewählt. Das ist eine translatorische Analysemethode, die bei der Rezeption des zu übersetzenden Textes angewendet wird. Der Text wird somit in einzelne Sinneinheiten intuitiv gegliedert. Die Größe einer Sinneinheit variiert laut Jurij Stepanov (1965: 258f.) nach Grenzen und Rhythmus des eigenen Verstehens in der Zeit. Eine Übersetzungseinheit ist somit der verstandene Textabschnitt bzw. die kontextuell oder sinngemäß erfasste Textebene, die bei gegebener Übersetzungsmethode übersetzt werden kann. Diese Größe der temporalen Differenz zwischen der Aufnahme des Originaltextes und der Produktion der Übersetzung, die als Übersetzungseinheit definiert wird, wird in der Referenzphase des Übersetzungsprozesses kognitiv festgestellt. Die Referenzphase ist nichts anderes als diese temporale Distanz selbst, die schwarze Box, in der das Verstehen bzw. mentales Übersetzen abläuft. Die kognitive Feststellung der Übersetzungseinheit 
erfolgt also in der Referenzphase, die Übersetzungseinheit entspricht somit in kognitiver Hinsicht der Referenzphase des Übersetzungsaktes selbst. Diese temporale Distanz bzw. Differenz ist jeder Translationsart eigen. Das Verhältnis des Originals zu seiner Übersetzung basiert auf einer Differenz, die aus der probabilistischen Natur des Verstehensprozesses entstanden und aus der Festlegung des notwendigen Differenzierungsgrads nach Paul Kußmaul und Hans G. Hönig (1982: 58) abzuleiten ist und den Verlauf des Übersetzungsprozesses prägt und gestaltet. Die Festlegung des notwendigen Differenzierungsgrads ergibt sich aus der Prämisse des lakunären Charakters der interkulturellen Kommunikation, deren Produkte literarische Texte sind. Die Differenz zeigt somit den Grad der Kongruenz der Verständigung zwischen verschiedenen Kulturen an. Der Ausgangspunkt für den erfolgreichen Verlauf der interkulturellen Kommunikation ist die Berücksichtigung der Differenz (Lakune), d.h. der kulturellen Spezifik. Sie gilt folglich als Ausgangspunkt für die Herstellung der Äquivalenzrelationen zwischen Original und Translat, zeichnet diese semantisch aus, was dem Postulat von „equivalence in difference" von Roman Jakobson entspricht. Sich auf den notwendigen Differenzierungsgrad festzulegen heißt nichts anderes als den Translationsakt zu vollbringen. Die Differenz erklärt den Übersetzungsprozess für möglich und notwendig. Sie ist sein eigentliches Charakteristikum. Die Differenz ist sowohl der Prozess des Übersetzens selbst als auch dessen Resultat. Die Berücksichtigung der Kulturspezifik ist Garant bzw. Unterpfand für den erfolgreichen Verlauf des Übersetzungsprozesses. Die Übersetzungseinheit ist daher eine kognitive Einheit des individuellen Denk- und Verstehensstils des Übersetzers. Ihre Grenzen hängen vom individuellen Fassungsvermögen eines jeden Übersetzers ab, sie liegt dem Übersetzungsakt zu Grunde. Die intuitive Feststellung von Übersetzungseinheiten basiert auf stochastischen implikativen Prozessen der Bedeutungsinterpretation.

Im zweiten Schritt wird das translatorische Experiment durchgeführt, das sich aus zwei Vorgehensweisen zusammensetzt - der Innenperspektive (introspektive bzw. prospektive Sicht) und der Außenperspektive (retrospektive Sicht auf den Übersetzungsprozess), dem das s.g. stilistische Experiment von Fëdorov (1970: 34) zu Grunde liegt (Panasiuk 2016: 231). Das stilistische Experiment gilt folglich als Analogie des Übersetzungsprozesses, das jeder statischen Kategorie bar ist. Die Dynamik des Übersetzungsprozesses, so wie sie im Bewusstsein eines Übersetzers abläuft und die Form von angenommenen und abgelehnten Varianten auf dem Papier annimmt, kann Fëdorov (ebd.) zufolge kaum mit Hilfe von Schemata und Modellen dargestellt werden, denn die Formen des Ablaufs dieses Prozesses sind unendlich verschieden. Die Anwendung des stilistischen Experiments kann folglich in zweierlei Hinsicht gesehen werden - in Bezug auf die Darstellung des Übersetzungsprozesses und auf die Bewertung der bereits vorhandenen Übersetzungen. Daher erweist sich die Durchführung der Analyse von Übersetzungen in zwei Richtungen als wichtig: Einerseits sind das verschiedene Übersetzungsvarianten des Originals, die von einem Übersetzer produziert wurden. Diese Vor- 
gehensweise ermöglicht es, durch die Anwendung des stilistischen Experiments den Übersetzungsprozess zu rekonstruieren. Und andererseits ist das die Analyse verschiedener Übersetzungen eines literarischen Textes, die von verschiedenen Übersetzern angefertigt wurden. Auf diesem Wege kann gezeigt werden, wie sich die Kreativität der Übersetzung sprachlich gestaltet bzw. erreicht sowie beschrieben werden kann. Die beiden Vorgehensweisen sind aufeinander bezogen, denn sowohl die erste als die zweite erlauben es, den Übersetzungsprozess intuitiv zu rekonstruieren. Die beiden Vorgehensweisen etablieren in ihrer Gesamtheit das translatorische Experiment. Das translatorische Experiment kann aus diesem Grunde sowohl als Übersetzungsprozess selbst als auch als analytische Methode betrachtet werden. Der Übersetzer vollzieht den Übersetzungsprozess, indem er unwissentlich das stilistische Experiment bzw. das kontextuell gebundene assoziative Experiment durchführt, das sich auf die probabilistischen Prozesse der Bedeutungsinterpretation gründet. Wie dies funktioniert, kann sowohl durch die Studie von Übersetzerkladden als auch durch mehrere Varianten einer Übersetzung nachvollzogen werden.

Dem translatorischen Experiment sollen weiterhin zwei deutsche Übersetzungen des Romans „Der Meister und Margarita“ von Michail Bulgakow sowie des Romans „Doktor Shiwago“" von Boris Pasternak unterzogen werden. Im Rahmen der Innenperspektive werden in diesem Zusammenhang zwei professionellen Übersetzern, die eigene Übersetzungsreflexion betreiben sollen, einige die Übersetzungseinheiten enthaltende Textabschnitte zum Übersetzen vorgeschlagen. Die Selbstreflexionen werden gleichzeitig auf einem Informationsträger aufgezeichnet. Die erhaltenen Aufnahmen werden anschließend in Form eines Protokolls des lauten Denkens angefertigt und analysiert. Die retrospektive Sicht auf den Übersetzungsprozess besteht darin, dass die Entsprechungen für die ausgewählten Übersetzungsvarianten, die in Bezug auf das Original sowie untereinander das Verhältnis eines translatorischen Feldes eingehen, in den zwei deutschen Übersetzungen der beiden Romane festgestellt werden. Die Ergebnisse der beiden Perspektiven werden anschließend miteinander verglichen. Der Vergleich der beiden Perspektiven soll die Rekonstruktion des tentativen Verlaufs des Übersetzungsprozesses im ersten Schritt hypothetisch ergeben.

Das translatorische Feld ist das Resultat des Übersetzungsprozesses, gilt als Erscheinung der Polyvarietät der Übersetzung, die sich auf die Relationen der intersprachlichen kontextuellen Synonymie gründet. Das Vorhandensein mehrerer sprachlicher Ausdrucksformen für einen bestimmten invarianten Inhalt versetzt den Übersetzer in eine Situation der Wahl bzw. der Entscheidung. Die Wirksamkeit der Synonymie im Bereich einer Sprachäußerung liegt nach Gauger (1972: 57) in ihrer Sprach-Bewusstheit. Dort, wo die Sprach-Bewusstheit eintritt, wird die Synonymie wirksam. Mit anderen Worten löst die Bewusstmachung einer Sprachäußerung den Prozess der Wahl von Synonymen aus, die die intendierte Sprachäußerung gemäß ihres Sinns verbalisieren. Die kognitive Bildung einer Sprachäußerung wird 
somit vermöge der Aktivierung der Synonyme, die für den Sinn dieser Sprachäußerung in Frage kommen, erreicht. Die kontextuelle Synonymie fußt wiederum auf den assoziativen Bedeutungen.

Dadurch erweist sich im dritten Schritt des psycholinguistischen Experiments der Einsatz des kontextuell gebundenen Assoziierens als sinnvoll. Das assoziative Experiment wird folglich mit den Übersetzungseinheiten des Originals, die als Stimuli fungieren, mit den anderen zwei professionellen Übersetzern durchgeführt. Die Ergebnisse des kontextuell gebundenen Assoziierens werden anschließend mit den translatorischen Feldern verglichen. Der tentative Verlauf des Übersetzungsprozesses kann dadurch hypothetisch rekonstruiert werden. Wichtig sei dabei die Tatsache bedacht, dass das mentale Lexikon eines erfahrenen Übersetzers immer mindestens zweisprachig strukturiert ist. Der Übersetzer denkt also mit den intersprachlichen semantischen Feldern (Semënova 1989: 37), denen der kognitive Mechanismus, das Relais des code-switching zu Grunde liegt (Min'jar-Beloručev 1996: 101ff.). Das intersprachliche semantische Feld setzt sich nur aus einer lexikalischen Einheit der Ausgangssprache und mehreren ihr entsprechenden Einheiten des Feldes der analogen Bezeichnung in der Zielsprache zusammen. Einer Originalbezeichnung entsprechen im Rahmen eines intersprachlichen semantischen Feldes mehrere Varianten, intersprachliche Synonyme für die Originalbezeichnung. In kommunikativer Hinsicht entsteht somit quasi ein Aufeinanderlegen von zwei Feldern derselben Bezeichnung der beiden Sprachen. Das zentrale Segment eines intersprachlichen Feldes setzt sich laut Semënova (ebd.) aus einer oder mehreren Einheiten des zielsprachlichen semantischen Feldes zusammen, die als Entsprechungen für die semantische Einheit des Originals am häufigsten auftreten. In der Struktur des intersprachlichen Feldes sieht Semënova ein mögliches Modell der translatorischen Entscheidungen. Die translatorische Entscheidung hängt nicht nur davon ab, zu welchem Segment des intersprachlichen Feldes die gewählte Variante gehört, sondern in erster Linie steht die funktionale (struktur-semantische, stilistische und pragmatische) Invarianz des Textes im Vordergrund. Das kann die Anwendung der Variante, die dem zentralen Segment des intersprachlichen Feldes am nächsten steht, für unerwünscht und unmöglich erklären. Bei der Arbeit mit dem intersprachlichen semantischen Feld ist es daher wichtig, die Logik der translatorischen Entscheidung zu ergründen und Faktoren aufzudecken, die den Übersetzer zwingen, von der Anwendung strukturell-analoger und semantisch-ähnlicher Einheiten abzusehen und die Varianten mit einer anderen syntaktischen und semantischen Struktur vorzuziehen. Dabei spielt die Intuition des Übersetzers eine große Rolle, mit deren Hilfe der Übersetzer die im Übersetzungsprozess vergleichenden Textabschnitte funktional abschätzt.

Das intersprachliche semantische Feld, das in jedem Übersetzungsakt aktiviert wird, stellt den Prozess der Suche nach Übersetzungsvarianten in der Verifizierungsphase, beim mentalen Übersetzen, dar. Die Existenz der intersprachlichen Felder sieht die Vielfältigkeit translatorischer Entscheidungen vor, denn den 
intersprachlichen Feldern ist die Vielfältigkeit der Ausdrucksweisen des ausgangssprachlichen Gedankenguts eigen. Die innere Struktur des mentalen Lexikons des Übersetzers setzt sich aus intersprachlichen semantischen Feldern zusammen, die durch den Mechanismus als translatorische Fertigkeit des Umschaltens (code-switching) eintrainiert werden.

Diese Tatsache rechtfertigt die Durchführung des kontextuell gebundenen assoziativen Experiments gerade mit den translatorischen Feldern. Assoziationen auf ein bestimmtes Stimulus-Wort, hier ein translatorisches Feld, werden in diesem Zusammenhang durch den Kontext determiniert. Derartige kontextuell gebundene Assoziationen sollen meistens rascher als im Fall eines freien Assoziierens erfolgen. Das sind paradigmatische Assoziationen, die der intersprachlichen kontextuellen Synonymie zu Grunde liegen. Der Kontext eines Wortes kann die Bedeutung dieses Wortes dadurch beeinflussen, dass durch den betreffenden Kontext bestimmte Assoziationen dieses Wortes wirksam werden, während die anderen in den Hintergrund treten und dadurch weniger verfügbar werden. Dem Übersetzer werden also die translatorischen Felder vorgelegt, zu denen er seine rezeptiven Reaktionen in Form von Responses (Assoziationen) aufschreiben soll, wobei diese sowohl ausgangs- als auch zielsprachlich bzw. beides sein können. Laut Deese (1962: 163) und Hörmann (1967: 183f.) wird die Bedeutung eines Wortes als Verteilung der Wort-Responses, die auf den betreffenden Stimulus hin produziert werden, definiert. Es wird somit ein Netz verbaler Assoziationen auf den Stimulus hin erzeugt, das als assoziatives Feld und gleichzeitig als Bedeutung des Schlüsselwortes bezeichnet werden kann. Bedeutung ist nach Hörmann (1967: 185) ein feldartiger Zusammenhang, den eine Palette von Assoziationen etabliert. Bedeutung als Zusammenhang sieht Hörmann in zweierlei Hinsicht als Zusammenhang des Wortes mit außersprachlichen Verhaltensereignissen sowie als Zusammenhang des Wortes mit anderen Wörtern, die seine verbale Umgebung etablieren, an. Die Bedeutung eines Wortes wird also in den Responses gesehen, die es auslöst. Deese (1962: 163) erfasst die assoziative Bedeutung eines Wortes dadurch, dass er die Relationen beschreibt, die zwischen den Responses auf verschiedene Stimuli bestehen: "If the associative meaning of any stimulus is given by the distribution of responses to that stimulus, then two stimuli may be said to have the same associative meaning when the distribution of associates to them is identical. Two stimuli overlap or resemble one another in associative meaning to the extent that they have the same distribution of associates“. Der Zusammenhang mit und zwischen den assoziativen Responses ist die assoziative Bedeutung des betreffenden Stimulus. Die Verteilung von Assoziationen zu dem zu untersuchenden translatorischen Feld soll über den hypothetischen bzw. möglichen Verlauf des Übersetzungsprozesses Aufschluss geben. Der assoziative Zusammenhang ermöglicht es schließlich, den weiteren Verlauf des Kontextes zu antizipieren, d.h. den Gesamtkontext zu erfassen. Die translatorischen Felder als Stimuli bieten gerade die Möglichkeit für das Erfassen der kontextuellen Bedeutung einer Sinneinheit. Der Übersetzer als Pro- 
band rekonstruiert somit die assoziativen Netzwerke des translatorischen Feldes. Die Ergebnisse des kontextuell gebundenen Assoziierens mit den translatorischen Feldern werden im dritten Schritt mit den Ergebnissen des kontextuell gebundenen Assoziierens im zweiten und denen der LD-Protokolle im ersten Schritt verglichen. Das soll das hypothetische Gesamtbild des Übersetzungsprozesses verschärfen.

Eine wichtige Rolle spielen dabei Emotionen. Die Untersuchung des Zusammenhangs zwischen Kognition und Affekt gilt als zentrale Problematik sowohl für die Untersuchung von kognitiven Prozessen als auch für die Untersuchung der Entwicklungsdynamik eines Menschen. Emotionen steigern die Varietät von Bedeutungen. Ins Spiel kommt hier der Einfluss der affektiven Färbung der Wörter auf die assoziativen Prozesse, denn unter der psychologischen Struktur einer Bedeutung wird ihre assoziative Struktur erkannt (Brudnyj 1971, A.A. Leont'ev 1971, Piaget 1963). Die Emotionen geben somit dem Denkprozess einen Kontext und eine Zielrichtung vor, welche die Komplexität des Denkprozesses reduzieren, d.h. den Denkprozess in richtige Bahnen lenken, ihm die kontextuelle Verbrämung verleihen. Die emotionale Bedeutung des Gedachten stellt das Denken vor allem in einen Beziehungskontext. Wird die Beziehungsgestaltung der Kognition durch strukturelle Störungen massiv beeinträchtigt, erlebt das formale Denken die Auswirkung von überflutenden Emotionen. Sollte die emotionale Erregung ihre Grenzen erreichen, die die Spaltungsprozesse verursacht, greifen die Letzteren auf die Kognition und gleichzeitig auf ihren sprachlichen Ausdruck über. Das zieht auch die fehlende Kohärenz der sprachlichen Aussagen nach sich.

Daniel Goleman (1997/2017: 49) sieht den emotiven Aspekt im neurowissenschaftlichen Zusammenhang. Die Emotionen verfügen demzufolge über eine Intelligenz. Das Wechselspiel von Gefühl (Emotionen) und Rationalität (Kognition) steuert das momentane Treffen einer Entscheidung eines Menschen. Die Emotionen können durch das denkende Gehirn entsprechend beeinflusst werden. Goleman (ebd.) betrachtet das komplementäre Verhältnis zwischen limbischem System und Neokortex, Mandelkern und Präfrontallappen als Instanzen, die durchaus am menschlichen Gefühlsleben teilhaben. Übertragen auf den Übersetzungsprozess ist es wichtig, bei der Selbstreflexion den Einfluss von Emotionen auf das Treffen einer Entscheidung für eine bestimmte Übersetzungsvariante zu beobachten. Wie verläuft der Übersetzungsprozess bei der Aufwallung von Emotionen? Ob der Prozess beschleunigt wird oder ins Stocken gerät, kann oft beim Dolmetschen in den polizeilichen Vernehmungen beobachtet werden. Oft kommen in solchen Situationen Momente vor, wenn Gemüter beiderseits zum Kochen von Emotionen gebracht werden. Dies schlägt sich in der Wahl der emotionsgeladenen Lexik nieder, die beim Dolmetschen wiedergegeben werden soll. Der emotionale Aspekt gibt den kontextuellen Rahmen vor, indem der Übersetzer versucht, die emotionale Ladung der Wörter in den betreffenden Sprachen und Kulturen wiederzugeben. Der emotionale Aspekt beschleunigt meistens die Suche nach prototypischen Kernvorstellungen innerhalb einer Szene, die die Form von kontextkonformen Über- 
setzungsvarianten annehmen. Die Findung der treffenden Übersetzungsvariante erleichtern die Emotionen, die die Assoziationskompetenz beim Übersetzer anregen. Die Emotionen sind mit einer bestimmten Situation verbunden, die lediglich in dieser Situation erlebt und erlitten und entsprechend dieser Situation sprachlich „gekleidet" werden. Die Emotionen rahmen die prototypischen Kernvorstellungen innerhalb einer Szene ein, indem sie ihnen den kontextuellen Rahmen, frame, vorgeben. Das kognitiv-neurowissenschaftliche Wissen über den Verlauf des Übersetzungsprozesses ermöglicht die bewusste Steuerung der translatorischen Tätigkeit durch einen Übersetzer. Die Vorstellung über die kognitiv-neurowissenschaftliche Organisation des Verstehens- und Übersetzungsprozesses wird zur Optimierung der translatorischen Tätigkeit beitragen. Der Einfluss von Emotionen auf die Kognition gilt unter Anwendung von psycholinguistischen Methoden experimentell noch zu klären und zu untersuchen.

\section{Literatur}

Anderson, John B. / Bower, Gordon (1973): Human associative memory. Washington.

Anderson, John B. (1976): Language, memory, and thouth. Hillsdale, NJ.

Annoni, Jean-Marie / Lee-Jahnke, Hannelore / Sturm, Annegret (2012): Neurocognitive Aspects of Translation. In: Meta 57, Nr. 1, S. 96-107. doi: 10.7202/1012743ar.

Brudnyj, Aron A. (1971): Značenie slova i psichologija protivopostavlenij. In: Leont'ev, Aleksej A. (Hrsg.): Semantičeskaja struktura slova. Psicholingvističeskie issledovanija. Moskva. S. 19-27.

Bulgakow, Michail (1975): Der Meister und Margarita. Berlin.

Černov, Gellij V. (1987): Eta mnogostradal'naja neperevodimaja „Alisa“ (implikativnost' i perevod). In: Gončarenko, Sergej F. (Hrsg.): Tetradi perevodčika. Naučno-teoretičeskij sbornik. Vypusk 22, S. 17-29.

Chernov, Ghelly (1990): On Sources of and Limits on Variability in Translation. In: Salevsky, Heidemarie (Hrsg.): Übersetzungswissenschaft und Sprachmittlerausbildung. Akten der I. Internationalen Konferenz, Berlin, 17.-19. Mai 1988, Bd. I, Humboldt-Universität zu Berlin. S. 132-134.

Deese, James (1962): On the structure of associative meaning. In: Psychological Review 69, S. 161175.

Deese, James (1965): The Structure of Associations in Language and Thought. Baltimore.

Eco, Umberto (1994): Lector in fabula. Die Mitarbeit der Interpretation in erzählenden Texten. München.

Ejger, Genrich V. (1990): Mechanizmy kontrolja jazykovogo vyskazyvanija. Char'kov.

Ekman, Paul (2010/2017): Gefühle lesen. Wie Sie Emotionen erkennen und richtig interpretieren. 2. Auflage. Berlin/Heidelberg.

Fëdorov, Andrej V. (1970): O sootnošenii edinogo i celogo v processe perevoda kak tvorčestva. In: Voprosy jazykoznanija 6, S. 27-35.

Gauger, Hans-Martin (1972): Zum Problem der Synonymie. Avec un résume en français: Apport au problèm des synonymes. Tübingen.

Graf, Richard (2018): Die neue Entscheidungskultur: Mit gemeinsam getragenen Entscheidungen zum Erfolg. München.

Goleman, Daniel (2007): EQ. Emotioniale Intelligenz. München. 
Gorlée, Dinda L. (2000): Der abduktive Ansatz in Übersetzungspraxis und Übersetzungsforschung. In: Wirth, Uwe (Hrsg.): Die Welt als Zeichen und Hypothese. Perspektiven des semiotischen Pragmatismus von Charles S. Peirce. Frankfurt. S. 158-180.

Gorlée, Dinda L. (2004): On Translating Signs. Exploring Text and Semio-Translation. Amsterdam/Atlanta.

Hansen, Gyde (2005): Experience and Emotion in Empirical Translation Research with Think-Aloud and Retrospection. In: Meta 50, Nr. 2, S. 511-521. doi:10.7202/010997ar.

Hönig, Hans G. / Kußmaul, Paul (1982): Strategie der Übersetzung. Ein Lehr- und Arbeitsbuch. Darmstadt.

Hönig, Hans G. (1995): Konstruktives Übersetzen, Bd. 1. Tübingen.

Hörmann, Hans (1967): Psychologie der Sprache. Berlin/Heidelberg/New York.

Jay, Timothy (2000): Why we curse. A Neuro-psycho-social Theory of Speech. Philadelphia/Amsterdam.

Kiss, George R. (1968): Words, associations, and networks. In: Journal of Learning and Verbal Behavior 7, S. 707-713.

Kiss, George R. (1969a): A computer model for certain classes of verbal behavior. In: Proceedings of the Inter national Joint Conference on Artificial Intelligence. Washington. S. 703-714.

Kiss, George R. (1969b): Steps towards a model of word selection. In: Machine Intelligence, Nr. 4.

Kiss, George R. (1973): An Associative Thesaurus of English: Structural analisis of a large relevance network, paper presented at the Conference on Current Research on Long-Term-Memory (Dundee, 1973). Edinburgh.

Kußmaul, Paul (2000): Kreatives Übersetzen. Tübingen.

Kußmaul, Paul (2007): Verstehen und Übersetzen. Ein Lehr- und Arbeitsbuch. Tübingen.

Leont'ev, Aleksej A. (1969): Psicholingvističeskie edinicy i poroždenie rečevogo vyskazyvanija. Moskva.

Leont'ev, Aleksej A. (1975): Psycholinguistische Einheiten und die Erzeugung sprachlicher Äußerungen. In deutscher Sprache herausgegeben von Fritz Jüttner. Berlin.

Min'jar-Beloručev, Rjurik K. (1996): Teorija i metody perevoda. Moskva.

Osgood, Charles E. (1980): Lectures on language performance (= Springer Series in Language and Communication, Bd. 7). New York.

Osgood, Charles E. / Suci, George J. / Tannenbaum, Percy H. (1957): The measurement od meaning. Urbana.

Panasiuk, Igor (2016): Polyvarietät der Übersetzung. Hamburg.

Pasternak, Boris (1995): Doktor Shiwago. Frankfurt am Main.

Piaget, Žan. / Inel'der, Barbel' (1963): Genezis elementarnych logičeskich struktur. Klassifikacii i seriacii. Perevod s francuyskogo E.M. Pčëlkinoj. Moskva.

Rudolf, Gerd (2012): Strukturbezogene Psychotherapie: Leitfaden zur psychodynamischen Therapie struktureller Störungen - Unter Mitarbeit von Hildegard Horn - Mit einem Geleitwort von Manfred Cierpka. Stuttgart.

Semënova, I.M. (1989): O ponjatii mežjazykovogo polja i vozmožnocti ego ispol'zovanija v lingvoperevodčeskicj issledovanijach. In: Černov, Gellij V. (Hrsg.): Kommunikativnyj invariant perevoda v tekstach različnych žanrov. Sbornik naučnych trudov. Vypusk 343. Moskova. S. 31-43.

Serson, Breno (2000): Semiotik und Kognitionswissenschaft. In: Wirth, Uwe (Hrsg.): Die Welt als Zeichen und Hypothese. Perspektiven des semiotischen Pragmatismus von Charles S. Peirce. Frankfurt am Main: Suhrkamp Taschenbuch Wissenschaft, S. 272-289.

Siever, Holger (2010): Übersetzen und Interpretation. Die Herausbildung der Übersetzungswissenschaft als eigenständige wissenschaftliche Disziplin im deutschen Sprachraum von 1960 bis 2000. Leipziger Studien zur angewandten Linguistik und Translatologie 8. Frankfurt am Main.

Germanica Wratislaviensia 145, 2020

(C) for this edition by CNS 
Stepanov, Jurij S. (1965/2002): Francuzskaja stilistika: v sravnenii s russkoj. La stylistique française. Vis-à-vis de la russe. Moskva.

Sun, Sanjun (2011): Think-Aloud-Based Translation Process Research: Some Methodological Considerations. In: Meta 56, Nr. 4, S. 928-951. doi: 10.7202/1011261ar.

Wilss, Wolfram (1992): Übersetzungsfertigkeit. Annäherungen an einen komplexen übersetzungspraktischen Begriff. Tübingen.

Wilss, Wolfram (1993): Überlegungen zum übersetzerischen Lehr- und Lernprozess. In: Holz-Mänttäri, Justa / Nord, Christiane (Hrsg.): Traducere Navem. Festschrift für Katharina Reiß zum 70. Geburtstag. Tampere. S. 103-116.

Zalevskaja, Aleksandra A. (1978): Voprosy organizacii leksikona čeloveka v lingviszičeskich i psichologičeskich issledovanijach: Učebnoe posobie. Kalinin.

Zalevskaja, Aleksandra A. (2005): Psicholingvističeskie issledovanija: Slovo. Tekst. Izbrannye tru$d y$. Moskva. 